\title{
MITRAGYNINE PERCENTAGES OF VARIOUS KRATOM VARIANTS SEIZED IN INDONESIA: A QUANTITATIVE ANALYSIS USING LIQUID CHROMATOGRAPHY-PHOTO DIODE ARRAY DETECTOR
}

\section{TANTI*, CHRISTIEN ANDRIYANI LALANGI, ERI ARFIYANI, WIDIANTI NINGTIAS, ERLANA NINDYA MAULIDA}

Quality and Research Division, the Center of Narcotics Laboratory of National Narcotics Board (BNN) of the Republic of Indonesia

${ }^{*}$ Email: tanti.puslabbnn@gmail.com

Received: 27 Apr 2021, Revised and Accepted: 19 Jun 2021

\section{ABSTRACT}

Objective: This study details the determination of mitragynine in various kratom samples using the thin-layer chromatography (TLC) technique and validation of analytical methods for quantifying the concentration of mitragynine in various kratom samples using liquid chromatography with photodiode array detector (LC-PDA).

Methods: TLC technique using n-hexane: ethyl acetate: ammonia 25\% (30: 15: $1 \mathrm{v} / \mathrm{v} / \mathrm{v}$ ) was applied to isolate mitragynine from kratom samples. Several interesting spots obtained were visualized under UV light at $254 \mathrm{~nm}$. Samples were also prepared with organic solvent extraction directly prior to LC analysis (non-isolation method of preparation) to quantify the concentration of mitragynine. Mobile phases used were acetonitrile (MP A) and 0.1\% formic acid in water (MP B). Samples and standards were run by gradient elution with a flow rate of $0.3 \mathrm{ml} / \mathrm{min}$, detection using PDA detector at $254 \mathrm{~nm}$.

Results: Mitragynine was successfully isolated from kratom samples in $\mathrm{R}_{\mathrm{f}} 0.50$ by TLC system applied. The validation of analytical methods for mitragynine passed the acceptance criteria as described by UNODC Guidance. The concentration of mitragynine in various kratom samples seized in Indonesia ranged from $0.37 \%-1.70 \%(\% \mathrm{w} / \mathrm{w})$.

Conclusion: Both TLC and LC analytical methods could be applied to determine and quantify the concentration of mitragynine in each examined sample, respectively.

Keywords: Kratom, Mitragynine, TLC technique, Validation, The concentration of mitragynine, LC, PDA detector

(C) 2021 The Authors. Published by Innovare Academic Sciences Pvt Ltd. This is an open access article under the CC BY license (https://creativecommons.org/licenses/by/4.0/)

DOI: https://dx.doi.org/10.22159/ijap.2021v13i5.41910. Journal homepage: https://innovareacademics.in/journals/index.php/ijap

\section{INTRODUCTION}

Kratom (Mitragyna speciosa Korth.) is widely cultivated in Indonesia especially in Kalimantan region. The plants are known as 'purik' or 'ketum' in West Kalimantan, 'kedamba/kedemba' in East Kalimantan and 'kayu sapat/sepat' in Middle and South Kalimantan. Among the region, West Kalimantan is the largest center of kratom plants in Indonesia. Kratom plants grow abundantly along the Kapuas river in Kapuas Hulu district. Products of kratom cultivated in Indonesia, $90 \%$ in the form of kratom powders, are mostly exported to other countries. The society in Kalimantan traditionally used kratom to combat fatigue and enhance stamina by drinking the brewed fresh kratom leaves or powder, sometimes by adding other ingredients such as honey or oranges [1].

The increasing reports of emergency rooms, hospitalizations and deaths related to kratom (mis)use in Western countries have been reported by $[2,3]$ as associated with the active ingredients of $M$. speciosa, mitragynine and 7-hydroxy mitragynine. Mitragynine and 7-hydroxymitragynine have an affinity towards opioid receptors and exhibit antinociceptive activity. Despite being a minor component of M. speciosa, 7-hydroxymitragynine was reported to have 46 times greater potency than mitragynine $[4,5]$. A growing number of kratom-related death reports were primarily in association with the abuse of other psychoactive substances. Mitragynine was reported in 27 cases as the cause of death and six of these case reports confirmed the presence of mitragynine and/or 7-hydroxy mitragynine solely as main autopsy findings [3].

Mitragynine belongs to indole-based alkaloid which is found as the major constituent of $M$. speciosa in Asian plants. The percentages of mitragynine are found in different levels depend on the age of the plants, the environment in where it is grown and how it is cultivated $[3,6]$. Kratom leaves originated from Indonesia contained the highest level of mitragynine and alkaloid fraction related to mitragynine than those from Southern Thailand and Northern Malaysia [7]. Different varieties, according to the color of kratom leaf veins e. g. red or green kratom and geography are associated with the diversity of alkaloid distribution in each plant and thus impact to its effect on users $[8,9]$.

To date, kratom plants and/or its main alkaloids are uncategorized as scheduled drugs in Indonesia. The previous analysis of kratom in The Center of Narcotics Laboratory was limited to the qualitative analysis using gas chromatography-tandem mass spectrometer (GC-MS). Due to the abundance of kratom plants in Indonesia, an exploration in kratom research is necessary to enrich data related to kratom in Indonesia. In the present study, we isolated mitragynine from various kratom powders in each specific label using a simple TLC technique followed by GC-MS analysis for determining the isolated compounds. We also measured the concentration of mitragynine from each sample without an isolation step before a quantitative analysis using LC-PDA.

The aims of this study were to determine the major alkaloid of $M$. speciosa, mitragynine, in various kratom samples seized in Indonesia using TLC technique and to quantify the concentration of mitragynine in the examined samples using an analytical method of LC-PDA that have been completely validated according to the UNODC Guidance [10]. The methods can hopefully be applied for screening kratom products in the drug market.

\section{MATERIALS AND METHODS}

\section{Materials}

Chloroform, n-hexane, ethyl acetate, ammonia solution $25 \%$, formic acid $98-100 \%$ and dichloromethane used were analytical grade. Acetonitrile, methanol and water used were chromatography grade. All solvents were purchased from Merck, Germany.

\section{Samples}

Samples analyzed in this study were six kinds of powders in transparent plastic bags with or without any specific labels. All samples were identified based on their physical characteristics such as: sample form, color and weight prior to further analysis. Description of each sample and sample identification are shown in table 1. 
Table 1: Physical characterization of each sample including the pictures of sample packaging (with the specific labels) and a unique sample code describing each sample (A-F)

\begin{tabular}{|c|c|c|c|c|}
\hline Sample code & Sample packaging with/without any specific labels & Form & Color & Net weight (g) \\
\hline A & & Fine powder & Olive green & 12.9000 \\
\hline B & & Fine powder & Olive green & 14.6000 \\
\hline $\mathrm{C}$ & & Fine powder & Olive green & 13.6000 \\
\hline D & & Fine powder & Army green & 11.4000 \\
\hline $\mathrm{E}$ & & Fine powder & Olive green & 13.7000 \\
\hline $\mathrm{F}$ & & Fine powder & Forest green & 16.7119 \\
\hline
\end{tabular}

Samples A-E were seized by BNN investigator on August 2019. Another case seized on October 2019 by BNN investigator was sample F. All samples were submitted to The Center of Narcotics Laboratory of BNN in Bogor, Indonesia, a few days after seized. Qualitative analysis using GC-MS was performed to all samples. Based on the result of analysis, samples A-F were found to contain mitragynine, an active alkaloid in kratom plant, so that called as kratom powders. Samples were subsequently transferred to the Research subdivision of The Center of Narcotics Laboratory of BNN for further analysis. This study began in early of 2020. Samples were kept in airtight vials and stored a refrigerator $\left(5-6^{\circ} \mathrm{C}\right)$ to avoid decomposition over the period of storage.

\section{Instrumentation}

Separation and determination of mitragynine in kratom powders were performed by TLC method on silica gel $60 \mathrm{~F}_{254} 10 \times 20 \mathrm{~cm}$ plates from Merck, Germany. CAMAG Linomat V was employed to spray varying amounts of extracted samples to the TLC plates. Spots of mitragynine were visualized under UV light at $254 \mathrm{~nm}$ using TLC Scanner from CAMAG, Switzerland.

Confirmatory analysis of mitragynine was carried out on GC-MS (EI) Agilent 7890B-5977B MSD. $1.0 \mu \mathrm{l}$ of the isolated compound was injected according to the split mode, the column was HP-5 MS UI 30 $\mathrm{m} \times 0.25 \mathrm{~mm} \times 0.25 \mu \mathrm{m}$. The oven temperature program was set as follows: $50{ }^{\circ} \mathrm{C}$ as initial temperature with $50{ }^{\circ} \mathrm{C} / \mathrm{min}$ to $200{ }^{\circ} \mathrm{C}$ (hold for $5 \mathrm{~min}$ ), followed by $10^{\circ} \mathrm{C} / \mathrm{min}$ to $300^{\circ} \mathrm{C}$ (hold for $10 \mathrm{~min}$ ). The injector and flow rate was $250{ }^{\circ} \mathrm{C}$ and $1 \mathrm{ml} / \mathrm{min}$, respectively. Total run time for each analysis was $40 \mathrm{~min}$.

Quantitative analysis of mitragynine was performed by UPLC-PDA Aquity H+Class (Waters, Singapore). $0.5 \mu \mathrm{l}$ of extracted samples were injected to LC, the column was Waters Acquity UPLC® BEH C18 $2.1 \times 100 \mathrm{~mm}, 1.7 \mu \mathrm{m}$ (wide bore). Mobile phase (MP) was acetonitrile (MP A) and $0.1 \%$ formic acid in water (MP B). Separation was achieved using the following MP gradient as follows: 0-1 $\mathrm{min}$ at $80 \%$ B decreasing to $50 \% \mathrm{~B}$ in $2 \mathrm{~min}$ (hold $1 \mathrm{~min}$ ), then increasing to $80 \%$ B in $4 \mathrm{~min}$. Flow rate was set on $0.3 \mathrm{ml} / \mathrm{min}$. Total run time needed for each injection was 8 min.

\section{Sample preparation}

There were two methods of preparation applied in this study, determination of mitragynine in kratom powders by isolation step and quantification of the mitragynine percentages without any isolation step (non-isolation method of preparation). Extraction of kratom powders was the very first step in both methods of preparation.

\section{Isolation method of preparation}

$200 \mathrm{mg}$ of homogenized samples were extracted with methanol: chloroform (80: 20) then vortexed for about 15 min followed by the filtration step. $1 \mathrm{ml}$ of each of the filtered solutions was evaporated using $\mathrm{N}_{2}$ gas. The residues were reconstituted with methanol prior to injection to GC-MS. It helped to understand the presence of mitragynine in the extracted samples before the isolation step was 
performed. To isolate mitragynine from kratom powders, varying amounts of extracted samples were sprayed on the TLC-activated plates. Spraying of the samples was repeated for 6 times in one plate to obtain 6 bands in starting line of the plates. Separation of mitragynine was achieved by n-hexane: ethyl acetate: ammonia $25 \%$ (30: 15: $1 \mathrm{v} / \mathrm{v} / \mathrm{v}$ ) [11]. Chamber was saturated for about $30 \mathrm{~min}$ at ambient temperature before the migration $[12,13]$. Plates were dried at room temperature after the migration. Spots were visualized under UV light at $254 \mathrm{~nm}$ using a TLC scanner. Layers of spots of interest were cut out from the plates then homogenized and weighed in three replicates for about $100 \mathrm{mg}$ to extract with methanol, followed by GC-MS analysis.

\section{Non-isolation method of preparation}

Method was modified from [14]. $12.5 \mathrm{mg}$ of homogenized samples were extracted with water: methanol (80:20) then vortexed and sonicated for 10-20 min. Extracted solutions were centrifuged under $6000 \mathrm{rpm}$. The supernatant of each sample was transferred to the 5 $\mathrm{ml}$ volumetric flask. Add methanol until the final volume was $5 \mathrm{ml}$. Extracted samples were filtered with PTFE filter prior to LC analysis. Extraction for all samples was carried out in three replicates.

\section{Preparation of standard solutions and method validation}

Validation of the analytical methods was carried out according to the UNODC Guidance [10]. Reference standard of mitragynine was purchased from Lipomed, Switzerland. A calibration curve of the standard solution was prepared by diluting subsequently the stock solution of mitragynine $100 \mu \mathrm{g} / \mathrm{ml}$ in methanol to six standard solutions in the range of $8.00-75.00 \mu \mathrm{g} / \mathrm{ml}$, each one in six replicates. A linear regression line equation and correlation coefficient (r) were constructed using the mean of peak areas versus concentrations of six standard solutions.

Accuracy was determined by preparing to reference standard solutions at the low, middle and high concentrations from the range of calibration curve concentration. The limit of detection (LOD), limit of quantification (LOQ) and precision were determined by dissolving varying amounts of non-isolated sample in methanol to obtain a stock solution of mitragynine $200 \mu \mathrm{g} / \mathrm{ml}$. LOD was measured by diluting stock solution until the lowest concentration that can be detected by the instrument. The peak of mitragynine should be identified in the sample which was shown by the similarity of both retention time and the wavelength to the standard. Repeatability and reproducibility conditions were measured to express the precision of the method. The specificity was investigated as well with AB-FUBINACA, a synthetic cannabinoid class, an example of controlled drugs from other groups.

\section{RESULTS AND DISCUSSION}

\section{Optimization of LC method}

For the best result of analysis, both isocratic and gradient elution were compared in this study. To evaluate the performance of both elution techniques, the reference standard of mitragynine was analyzed using a mobile phase of acetonitrile: formic acid $0.1 \%$ in water and the flow rate was $0.3 \mathrm{ml} / \mathrm{min}$. With PDA detection at 254 $\mathrm{nm}$, mitragynine was detected too early in 0.968 min by isocratic elution. A better separation was achieved by gradient elution where the peak of mitragynine was confirmed at $3.162 \mathrm{~min}$. Henceforward, the gradient elution was applied in the quantitative analysis of mitragynine using LC-PDA.

\section{Analytical method of validation}

A calibration curve was linear over the concentration range from 8.00$75.00 \mu \mathrm{g} / \mathrm{ml}$ with the regression line equation of $\mathrm{y}=8379.6490 \mathrm{x}-$ 775.6190. Limit of detection value was $0.4721 \mu \mathrm{g} / \mathrm{ml}$. All tested validation parameters satisfied the acceptance criteria as validation requirements. Results of method validation are summarized in table 2 .

\section{Isolation method of preparation}

Extraction and isolation of mitragynine from kratom leaves could be the most important step prior to any analysis scheme because of the complexity of plant chemical constituents [15]. Isolation of mitragynine was performed on all examined samples. Preliminary identification using GC-MS confirmed the presence of mitragynine in each of extracted samples. Fig. 1 shows the result of separation by TLC technique under the TLC Scanner from sample $F$ as representative. All samples exhibited similar profile of TLC chromatogram to F. Several layers of spots of interest were cut out from the plates to perform GC analysis. Upon the previous study of [11], the spots at $R_{f} 0.50$ were suspected to be mitragynine.

As the result of this study, mitragynine was successfully isolated from the spot at $R_{f} 0.50$ by a developed TLC system. GC analysis showed that isolation of kratom powders resulted in a single peak of mitragynine, and thus differs from the multi peaks of extracted sample chromatograms before the isolation (fig. 2 (B1-C1)). Peaks of mitragynine in extracted and isolated samples have a similar retention time to the standard of mitragynine, 33.407 min (fig. 2 (A1)).

Table 2: Summary of analytical method validation of mitragynine in kratom powders using LC-PDA

\begin{tabular}{ll}
\hline Parameters & Results \\
\hline Linearity and working range & $\mathrm{r}=0.999$ \\
LOQ $(\mu \mathrm{g} / \mathrm{ml})$ & 0.9782 \\
Precision under repeatability and & Intra-day = 1.67; Inter-day = \\
reproducibility conditions (\%RSD) & $1.62^{*}$ \\
Accuracy (\%RSD) & $1.644^{*}$ \\
Recovery (\%) & 99.57 \\
Specificity/Selectivity & 30.15 \\
Uncertainty & 0.47 \\
\hline
\end{tabular}

${ }^{*} \%$ RSD for the lower concentration

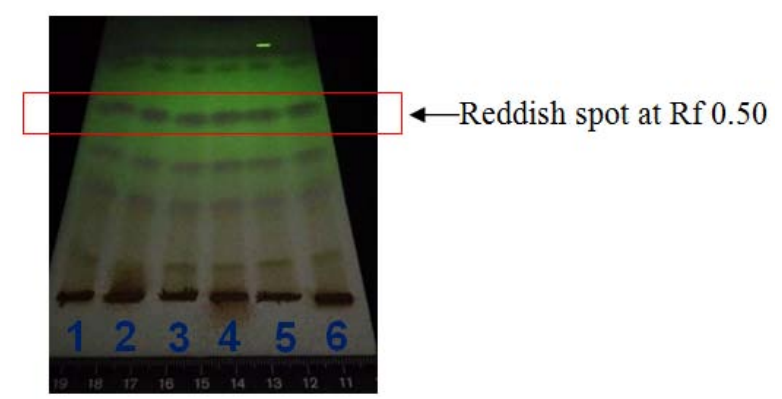

Fig. 1: TLC chromatogram of the extracted sample F under 254 nm wavelength, spots no. 2-6 were replicated to spot no. 1 in equal volume of each spot

In the right panel, fig. 2 (A2-C2) details the MS spectra of the reference standard of mitragynine, an extracted and isolated compound of sample $\mathbf{F}$, respectively. Mitragynine had fragmentation ion at $\mathrm{m} / \mathrm{z} 214.1$ (as base peak) followed by $\mathrm{m} / \mathrm{z} 397.2$, a similar finding to the previous study by [16]. Other fragment ions obtained were $\mathrm{m} / \mathrm{z}$ 186.1, m/z 199.1, m/z 269.1, m/z 281.1 and m/z 383.2.

\section{Non-isolation method of preparation}

A simple preparation was applied to the samples A-F to do a quantitative analysis using LC-PDA. Extract samples in water: methanol (80:20) were directly injected to the instrument without any isolation steps before. LC analysis of the samples exhibited a multi-peak on the resulting chromatogram, indicated that there were several compounds detected other than mitragynine. Therefore, the reference standard of mitragynine was used to identify the peak of mitragynine in kratom samples. Mitragynine was identified at approximately $3.117 \mathrm{~min}$ and 3.097-3.107 $\mathrm{min}$ in the standard and samples, respectively. There was a very slight retention time shift between standard and samples. It probably due to the column overloaded or changes in the mobile phase for each analysis [17]. However, the differences of retention time between standard and samples were less than $2 \%$ and thus meet the criteria of acceptance as described by [10]. Furthermore, all samples were confirmed as mitragynine by the similarity of the maximum 
wavelength $\left(\lambda_{\max }\right)$ to the reference standard of mitragynine which was $222.3 \mathrm{~nm}$ (fig. 3). Two other peaks at retention time of $\pm 3.15 \mathrm{~min}$ and $\pm 3.21 \mathrm{~min}$ in samples $\mathbf{A}-\mathbf{F}$ have the maximum wavelength $\left(\lambda_{\max }\right)$
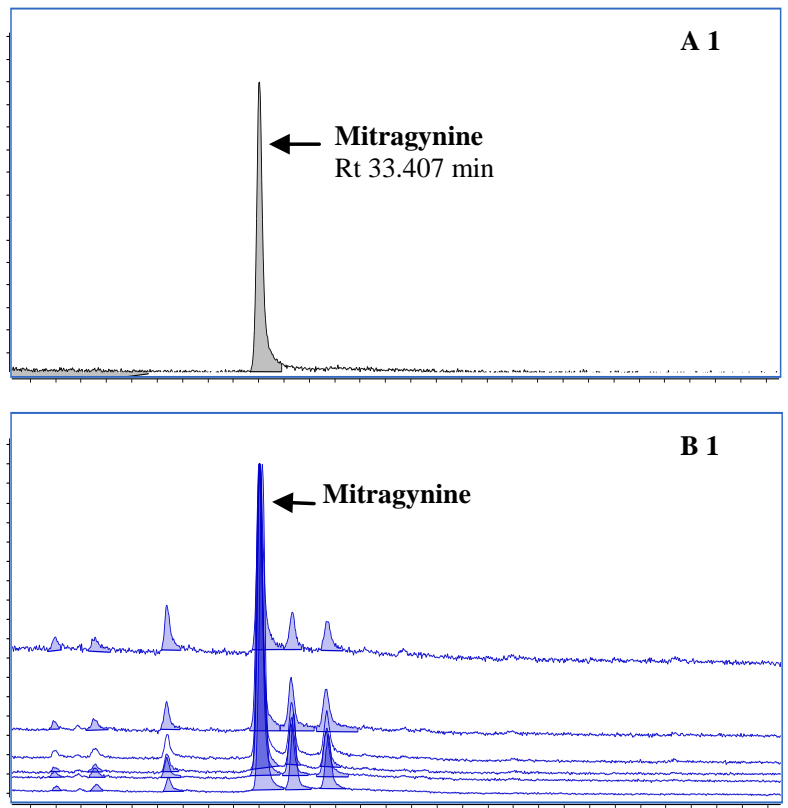

C 1

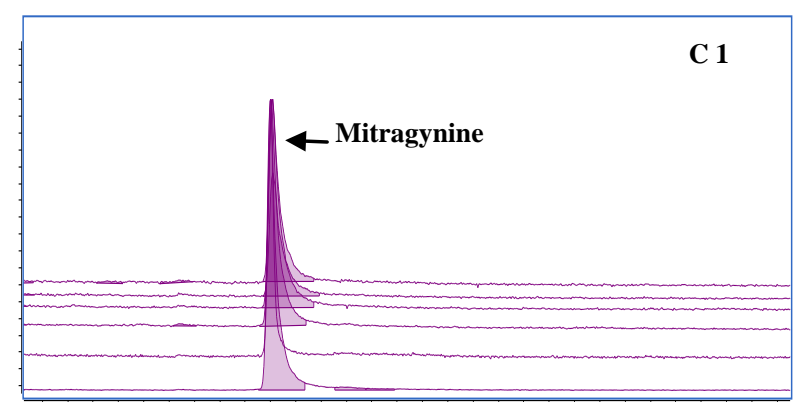

at $222.3 \mathrm{~nm}$. However, the first peak of each sample, which was the highest peak, was supposed to be mitragynine when compared to the standard.
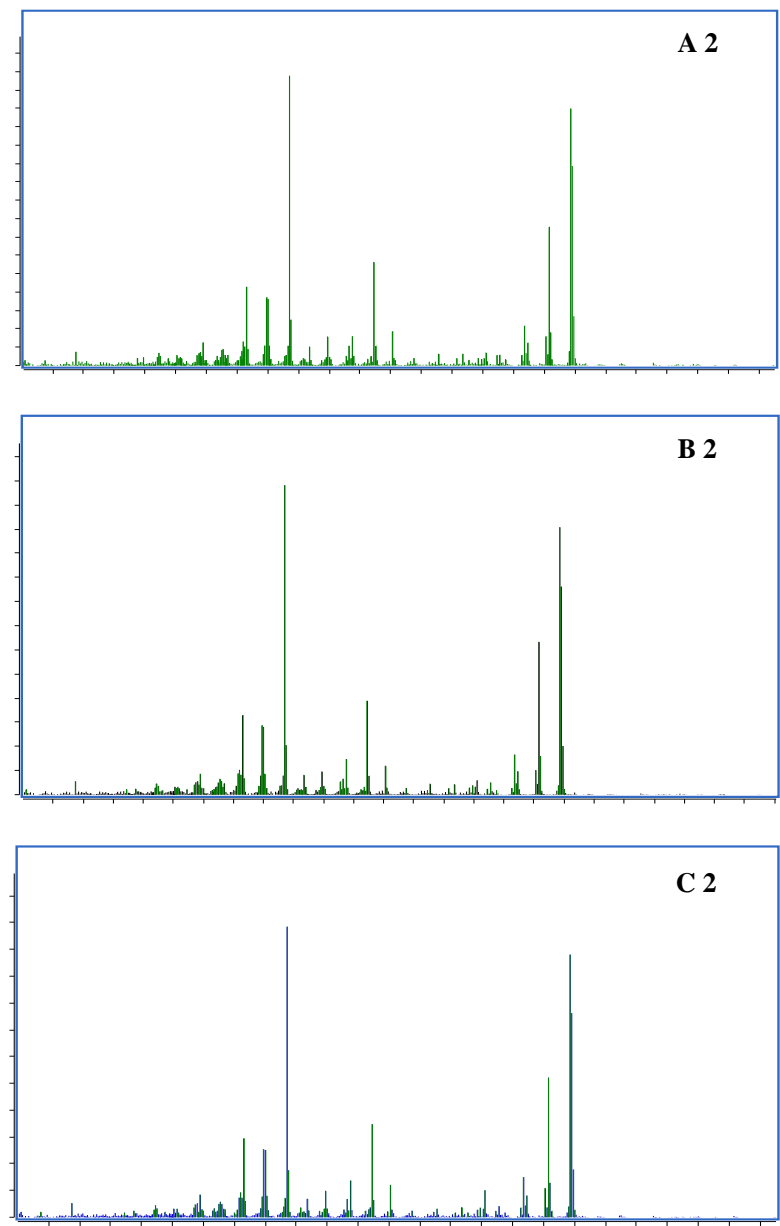

Fig. 2: Left panel, (A1) GC chromatogram of $100 \mu \mathrm{g} / \mathrm{ml}$ standard of mitragynine; overlayed GC chromatogram of (B1) extracted samples A-F in methanol: chloroform (80: 20) and (C1) isolated compound of samples A-F by TLC system at Rf 0.50. Right panel: MS fragment spectra of mitragynine of (A2) $100 \mu \mathrm{g} / \mathrm{ml}$ standard; (B2) extracted sample F as representative; (C2) isolated compound of sample F as representative

(a)

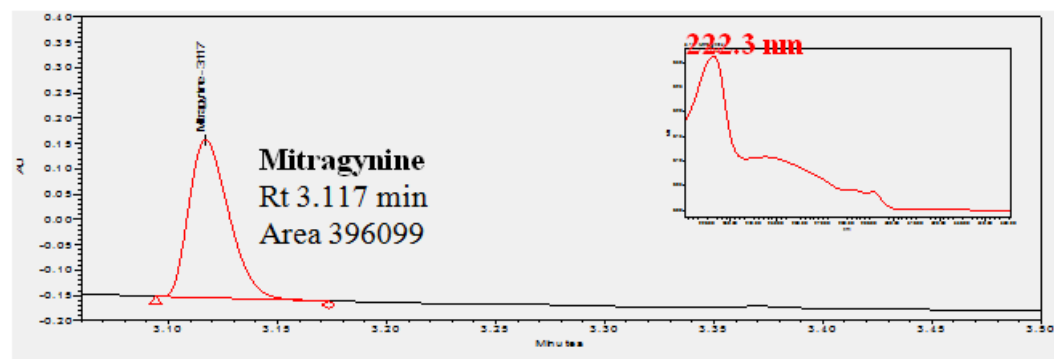

(b)

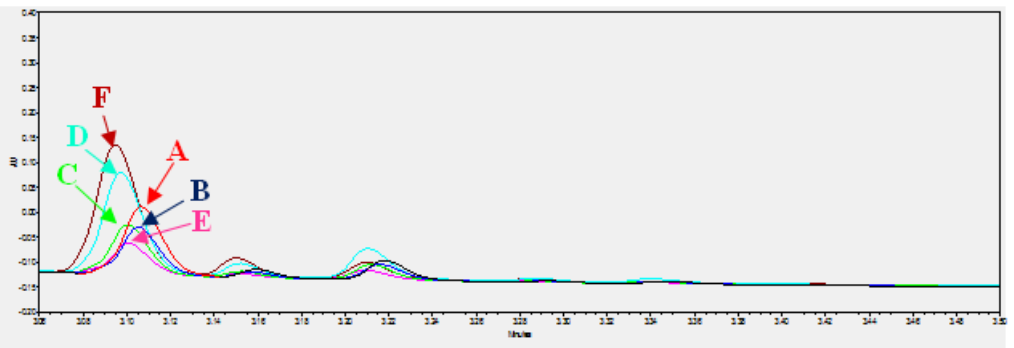

Fig. 3: LC chromatogram and PDA UV/Vis spectrum of mitragynine in (a) Reference standard of mitragynine $50 \mu \mathrm{g} / \mathrm{ml} \mathrm{and} \mathrm{(b)} \mathrm{samples} \mathrm{A-F}$ 
The concentration of mitragynine in each sample was measured by plotting the mean areas of mitragynine to the calibration curve of $y=$ $8379.6490 \mathrm{x}-775.6190$. Weight by weight percentage $(\% \mathrm{w} / \mathrm{w})$ of mitragynine was calculated with the following formula. Results of the analysis were shown in table 3.

$\frac{\text { (Area of mitragynine } \mathrm{x} \text { Final volume of sample } \mathrm{x} \text { Dilution factor) }}{\text { (Sample weight x 1000) }} \times 100 \%$

Table 3: Quantitative analysis of mitragynine in various kratom powders

\begin{tabular}{lll}
\hline Samples & $\begin{array}{l}\text { Quantity of mitragynine } \\
(\boldsymbol{\mu g} / \mathbf{m l})\end{array}$ & $\begin{array}{l}\text { Quantity of mitragynine } \\
(\% \mathbf{w} / \mathbf{w})\end{array}$ \\
\hline A & $20.6842 \pm 0.47$ & 0.83 \\
B & $14.2177 \pm 0.47$ & 0.57 \\
C & $15.1779 \pm 0.47$ & 0.61 \\
D & $31.3860 \pm 0.47$ & 1.25 \\
E & $9.2859 \pm 0.47$ & 0.37 \\
F & $42.4476 \pm 0.47$ & 1.70 \\
\hline
\end{tabular}

The concentration of mitragynine in samples A-F ranged from $0.37 \%-1.70 \%$ (table 3 ). In this study, kratom sample in the transparent plastic bag without any specific label (sample F) had the highest mitragynine concentration among others, followed by 'Green' kratom label (sample D). The lowest concentration of mitragynine was detected in the product of 'Yellow' label (sample E). The data show that kratom variants have an influence on the distribution of alkaloids in kratom plants, in this case was mitragynine. All samples have a lower concentration of mitragynine than kratom powders studied by [5]. Nowadays, sales of kratom products in many different types of kratom strains are very massive on the internet with no guarantee of the products purity as well as the concentration of mitragynine nor other alkaloids present in the products. The prices of each product are different to each other depends on the strains or varieties of kratom. The differences in the concentration of mitragynine are likely having an impact on the quality and potency though the effects of kratom used are relying on the dosage and also the administration of kratom consumption whether it is used solely or in combination with other psychoactive drugs.

\section{CONCLUSION}

In this study, extraction of methanol: chloroform (80: 20) was established prior to isolation of mitragynine from various kratom powders by TLC system. Mitragynine was detected from the layer of spots at $\mathrm{R}_{\mathrm{f}} 0.50$ in confirmatory analysis using GC-MS. For quantitative analysis, gradient elution was chosen for LC separation. The analytical method of LC-PDA was linear at the concentration range of $8.00-75.00 \mu \mathrm{g} / \mathrm{ml}$ with $\mathrm{r}=0.999$. Other validation parameters tested satisfied the requirements and thus valid for measuring the actual concentration of mitragynine in kratom samples. The concentration of mitragynine in various kratom variants seized in Indonesia varied from $0.37 \%-1.70 \%(\% \mathrm{w} / \mathrm{w})$.

\section{ACKNOWLEDGEMENT}

The authors would like to acknowledge the Center of Narcotics Laboratory of BNN, Republic of Indonesia for facilitating this study and for the BNN investigators who submitted kratom samples to our Lab.

\section{FUNDING}

Nil

\section{AUTHORS CONTRIBUTIONS}

$\mathrm{T}$ and CAL developed the concept of the study and supervised the study. EA performed the method validation. WN conducted the study including sample preparation, sample isolation and quantitative analysis. EA and WN analyzed and interpreted the data. ENM contributed in writing the manuscript. All authors agreed to the final version of this manuscript.

\section{CONFLICTS OF INTERESTS}

The authors have no conflicts of interest.

\section{REFERENCES}

1. Wahyono S, Widowati L, Handayani L, Sampurno OD, Haryanti S, Fauzi, et al. Kratom: health and socio-economic prospects. Jakarta: Publisher of National Institute of Health Research and Development; 2019. Available from: http://repository. litbang.kemkes.go.id/id/eprint/3869. [Last accessed on $06 \mathrm{Apr}$ 2021].

2. Chien GCC, Odonkor C, Amorapanth P. Is kratom the new 'legal high' on the block?: the case of an emerging opioid receptor agonist with substance abuse potential. Pain Phys 2017;20: E195-8.

3. Corkery JM, Streete P, Claridge H, Goodair C, Papanti D, Orsolini $\mathrm{L}$, et al. Characteristics of deaths associated with kratom use. J Psychopharmacol 2019;33:1102-23.

4. Takayama H. Chemistry and pharmacology of analgesic indole alkaloids from the rubiaceous plant, Mitragyna speciosa. Chem Pharm Bull 2004;52:916-28.

5. Kikura Hanajiri R, Kawamura M, Maruyama T, Kitajima M, Takayama H, Goda Y. Simultaneous analysis of mitragynine, 7hydroxymitragynine, and other alkaloids in the psychotropic plant "kratom" (Mitragyna speciosa) by LC-ESI-MS. Forensic Toxicol 2009;27:67-74.

6. Cinosi E, Martinotti G, Simonato P, Singh D, Demetrovics Z, Roman Urrestarazu A, et al. Following "the roots" of kratom (Mitragyna speciosa): the evolution of an enhancer from a traditional use to increase work and productivity in Southeast Asia to a recreational psychoactive drug in western countries. Biomed Res Int 2015;2015:1-11.

7. Orio L, Alexandru L, Cravotto G, Mantegna S, Barge A. UAE, MAE, SFE-CO2 and classical methods for the extraction of Mitragyna speciosa leaves. Ultrason Sonochem 2012;19:591-5.

8. Boffa L, Ghe C, Barge A, Muccioli G, Cravotto G. Alkaloid profiles and activity in different Mitragyna speciosa strains. Nat Prod Commun 2018;13:1111-6.

9. Chittrakarn S, Penjamras P, Keawpradub N. Quantitative analysis of mitragynine, codeine, caffeine, chlorpheniramine and phenylephrine in a kratom (Mitragyna speciosa Korth.) cocktail using high-performance liquid chromatography. Forensic Sci Int 2012;217:81-6.

10. Laboratory and Scientific Section, United Nations Office on Drugs and Crime. Guidance for the validation of analytical methodology and calibration of equipment used for testing of illicit drugs in seized materials and biological specimens. New York: United Nations; 2009.

11. Kowalczuk AP, Lozak A, Zjawiony JK. Comprehensive methodology for identification of kratom in police laboratories. Forensic Sci Int 2013;233:238-43.

12. Padh H, Parmar S, Patel B. Development and validation of stability-indicating HPTLC method for estimation of swertiamarin in bulk and dosage form. Int J Appl Pharm 2017;9:80-4

13. Gurupriya S, Cathrine L, Pratheema P. HPTLC method for the determination of lupeol from Andrographis echioides leaves. Int J Pharm Pharm Sci 2018;10:102-7.

14. Casey CR, Conley T, Heise A, Thomas T, Ayres PR. Quantitative and qualitative analysis of mitragynine in kratom (Mitragyna speciosa) by GC-MS, LC-MS/MS and UPLC-PDA. J Reg Sci 2015;2:1-14.

15. Jeyaleela GD, Monisha SI, Vimala JR, Immaculate AA. Isolation of 2-chlorobenzimidazole from Melia dubia leaf extract and its structural characterization. Int J Pharm Pharm Sci 2017;9:6772.

16. Chan KB, Pakiam C, Rahim RA. Psychoactive plant abuse: the identification of mitragynine in ketum and in ketum preparations. Bull Narc 2005;57:249-56.

17. Mcnair H, Polite LN. Troubleshooting in high-performance liquid chromatography. Sep Sci Technol 2007;8:459-77. 